ARTICLE OPEN

\title{
Articular cartilage and sternal fibrocartilage respond differently to extended microgravity
}

\author{
Jamie Fitzgerald (D) $^{1,2}$, Jamie Endicott ${ }^{1}$, Uwe Hansen ${ }^{3}$ and Cathleen Janowitz ${ }^{2}$
}

The effects of spaceflight on cartilaginous structure are largely unknown. To address this deficiency, articular cartilage (AC) and sternal cartilage (SC) from mice exposed to 30 days of microgravity on the BION-M1 craft were investigated for pathological changes. The flight AC showed some evidence of degradation at the tissue level with loss of proteoglycan staining and a reduction in mRNA expression of mechano-responsive and structural cartilage matrix proteins compared to non-flight controls. These data suggest that degradative changes are underway in the AC extracellular matrix exposed to microgravity. In contrast, there was no evidence of cartilage breakdown in SC flight samples and the gene expression profile was distinct from that of AC with a reduction in metalloproteinase gene transcription. Since the two cartilages respond differently to microgravity we propose that each is tuned to the biomechanical environments in which they are normally maintained. That is, the differences between magnitude of normal terrestrial loading and the unloading of microgravity dictates the tissue response. Weight-bearing articular cartilage, but not minimally loaded sternal fibrocartilage, is negatively affected by the unloading of microgravity. We speculate that the maintenance of physiological loading on AC during spaceflight will minimize AC damage.

npj Microgravity (2019)5:3; https://doi.org/10.1038/s41526-019-0063-6

\section{INTRODUCTION}

The major load-bearing tissue within the joint is articular cartilage (AC). AC is exquisitely sensitive to changes in biomechanical loading (reviewed by Sanchez-Adams et al. ${ }^{1}$ ). Under normal conditions, chondrocytes synthesize a balance of extracellular matrix (ECM) components so that the ability to resist tensile and compressive forces is maintained. Deviation from the normal range of biomechanical forces, ${ }^{2-5}$ including complete unloading, ${ }^{6-8}$ tips the balance from maintenance to pathology typically leading to cartilage erosion and later osteoarthritis. While the effects of the biomechanical unloading environment of spaceflight on bone and skeletal muscle are well-studied, ${ }^{9,10}$ the effects on AC are largely unknown. The responses of $A C$ to microgravity are important to define because it is clear from clinical studies that load-bearing AC is different from bone and skeletal tissue in that has a very poor capacity to restore damaged tissue. ${ }^{11}$ Consequently, microgravityinduced joint pathology could compromise flight crew mobility, interfere with mission activities, and accelerate short- and longterm joint degradation in flight personnel.

To investigate the effect of extended microgravity on $A C$, joint tissue from mice exposed to 30 days of Spaceflight in the BION-M1 flight was assessed for evidence of cartilage degradation. Our data suggest that spaceflight results in tissue degradation in loadbearing $A C$, but not in minimally loaded sternal fibrocartilage.

\section{RESULTS}

Articular cartilage

Spaceflight (SF) AC samples demonstrated less proteoglycan compared to AC ground controls (GC) (Fig. 1a). Decreased proteoglycan levels were generally restricted to the femoral condyle rather than the tibial plateau. Analysis of the boxed regions drawn around areas of reduced proteoglycan staining indicates approximately $35 \%$ of chondrocytes within the box stain for pericellular proteoglycan. Virtually no superficial zone chondrocytes have pericellular staining with the majority of toluidine blue-stained cells residing in the middle zone. Collagen II levels were similar between SF and control samples although three SF samples showed evidence of surface damage (Fig. 1b). In addition, clear evidence of osteophyte formation was present in three SF samples and in one GC and one SFV control sample (Fig. 1c). One SF femur sample was $<2 / 3$ the thickness of the average GC thickness. The SF mice had a significantly worse overall histological scores compared to all of the non-flight control groups, suggesting more overall cartilage degradation (Fig. 1d). Electron microscopy analysis of AC of elbows revealed no major differences in matrix density between SF and GC samples (Supplementary Fig. 1). Gene expression analysis showed several sustained changes in gene activation in SF compared to GC samples. Ten genes were upregulated and 37 genes downregulated greater than two-fold in flight compared to ground with a false discovery rate (FDR) of 0.05 (Fig. 2a). Seventeen of the altered genes are structural cartilage ECM proteins or proteins associated with joint pathology.

\section{Sternal cartilage}

The availability of sternal tissue allowed us to compare the response of the two cartilages to microgravity. Representative images of the same cartilaginous sternocostal synchondrosis in the region between incoming ribs and ossified sternebrae are

\footnotetext{
${ }^{1}$ Bone and Joint Center, Department of Orthopedic Surgery, Henry Ford Hospital System, Detroit, MI 48202, USA; ${ }^{2}$ Department of Orthopaedics and Rehabilitation, Oregon Health and Science University, Portland, OR 97239, USA and ${ }^{3}$ Institute of Musculoskeletal Medicine (IMM), University Hospital of Münster, 48149 Münster, Germany Correspondence: Jamie Fitzgerald (jitzge2@hfhs.org)
}

Received: 30 October 2017 Accepted: 31 January 2019

Published online: 18 February 2019 


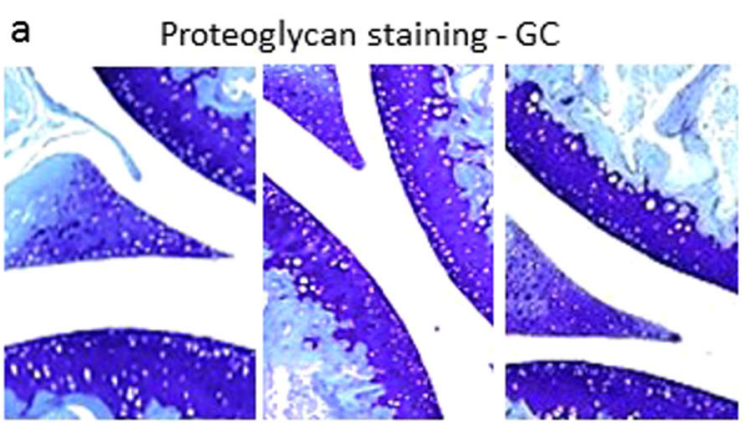

SF
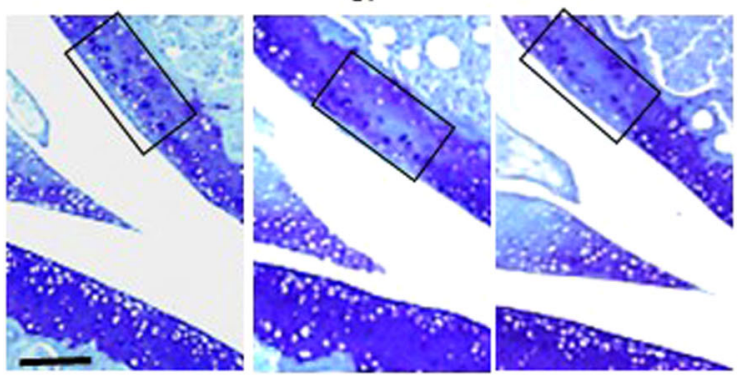

d
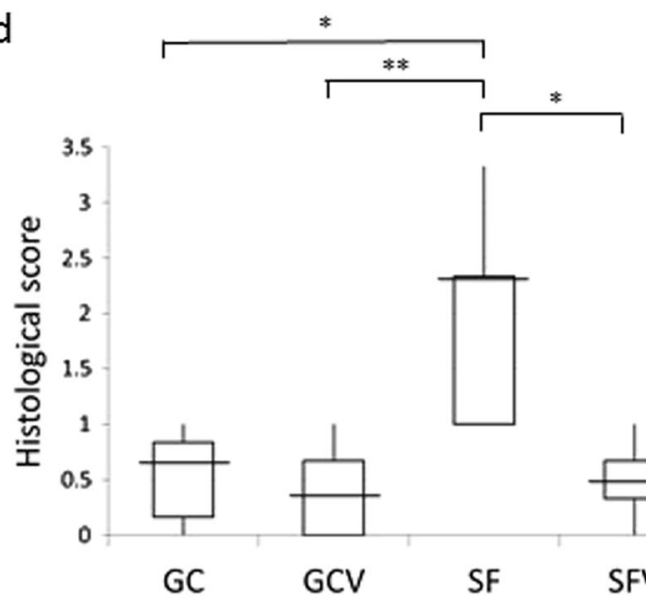
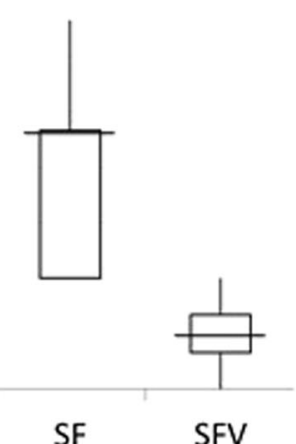

b Surface damage - SF
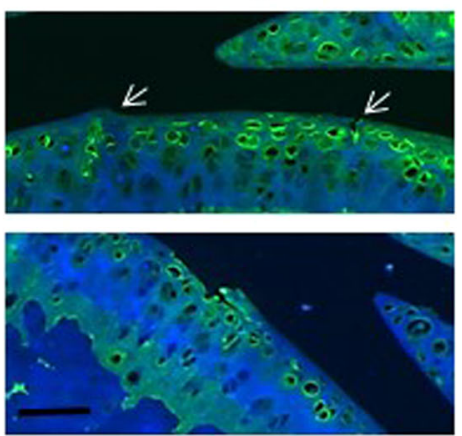

C Osteophyte formation - SF

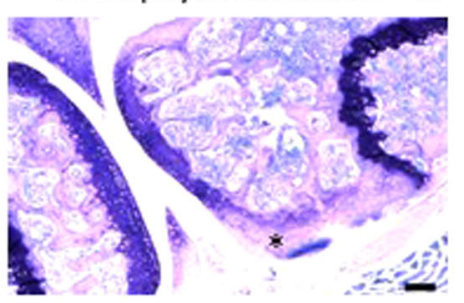

e

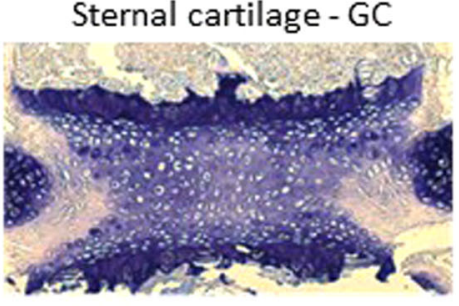

SF

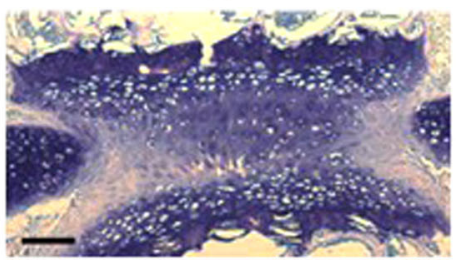

Fig. 1 Histological analysis of spaceflight cartilage. a Proteoglycan analysis of articular cartilage. Sagittal sections of femoro-tibial joint were stained with toluidine blue for proteoglycan. The boxed region defines the area used to calculate the proportion of chondrocytes exhibiting pericellular proteoglycan in regions of reduced territorial proteoglycan. Scale bar is $100 \mu \mathrm{M}$. b Superficial zone damage. Sections were stained for collagen II. Several sections had surface irregularities. Note the uneven surface and fissure in the top panel (arrows) and damaged surface layer in the lower panel. Scale bar is $50 \mu \mathrm{M}$. c Presence of osteophytes. Representative image from a toluidine blue-stained SF sample showing evidence of an osteophyte (indicated by an asterisk) on the femoral condyle. Scale bar is $100 \mu \mathrm{M}$. d Histological scores for SF and non-flight controls (GC, GCV, and SFV). SF samples have a significantly higher histological score compared to ground and vivarium controls using the Kruskal-Wallis test $\left({ }^{*} P<0.05,{ }^{*} P<0.01\right)$. Median, maximum score, minimum score, and 25 th and 75 th percentile of total histology data are plotted for each experimental group. Scoring matrix is shown in Supplementary Table 1 and $P$-values for all pairwise combinations of experimental group histological scores shown in Supplementary Table 2. e Proteoglycan analysis of sternal cartilage. Representative sections of sternum from SF and GC mice cut in the coronal plane were stained for proteoglycan. The images show a single cartilaginous sternocostal synchondrosis flanked by the incoming ribs and the bony sternebrae above and below. Growth plate chondrocytes are located adjacent to the zones of calcified cartilage. Scale bar is $100 \mu \mathrm{M}$

shown in Fig. 1e. This tissue is cartilaginous because it expresses the cartilage-specific gene Col10a1 according to microarray gene expression data (not shown). In contrast to AC, there was no difference in extent and overall levels of proteoglycan staining between SF and GC samples in the sternal cartilage (SC), indicating no microgravity-induced proteoglycan decrease. Gene expression analyses demonstrated that 30 sternal genes were upregulated and 35 were downregulated in SF compared to GC (Fig. 2b).

\section{DISCUSSION}

The finding of reduced proteoglycan levels in $A C$ due to microgravity is consistent with hindlimb unloading and limb immobilization studies in rodents, dogs, and rabbits, which consistently report unloading-specific $A C$ atrophy in multiple experimental situations (reviewed in ref. ${ }^{8}$ ). Our data suggest that despite partial proteoglycan reduction, the collagen II network remains intact. While surface damage and osteophytes were noted in several SF animals, there was no evidence of widespread 
a

\begin{tabular}{|c|c|c|}
\hline gene & flight/ground & description \\
\hline Igkv6-20 & 3.38 & immunoglobulin \\
\hline Eif3m & 2.53 & translation initiation factor \\
\hline Stfa2 & 2.12 & protease inhibitor \\
\hline Ms4a3 & 2.10 & macrophage cell surface receptor \\
\hline Gstm2 & 2.09 & glutathionine S-transferase \\
\hline Gm10417 & 2.06 & unknown \\
\hline Top2a & 2.06 & DNA topoisomerase \\
\hline Rbm3 & 2.05 & RNA-binding protein \\
\hline Ifitm6 & 2.01 & interferon-response gene \\
\hline Igkv4-91 & 2.00 & immunoglobulin \\
\hline Omd & -2.00 & ECM protein \\
\hline Olfr1437 & -2.02 & olfactory receptor \\
\hline Gm6432 & -2.02 & unknown \\
\hline Fmod & -2.04 & ECM proteoglycan \\
\hline Gm10673 & -2.04 & unknown \\
\hline Ogn & -2.04 & ECM protein \\
\hline C1s & -2.06 & serine protease \\
\hline Olfr118 & -2.07 & olfactory receptor \\
\hline Olfr1454 & -2.07 & olfactory receptor \\
\hline Serpina1b & -2.07 & protease inhibitor \\
\hline Olfr764 & -2.07 & olfactory receptor \\
\hline Gsn & -2.08 & actin binding protein \\
\hline Ccdc80 & -2.10 & adipogenesis inhibitor \\
\hline Slc35e3 & -2.11 & unknown \\
\hline Olfr948 & -2.14 & olfactory receptor \\
\hline Clu & -2.15 & complement-assoc protein \\
\hline Den & -2.18 & ECM protein \\
\hline Olfr347 & -2.18 & olfactory receptor \\
\hline Olfr1014 & -2.21 & olfactory receptor \\
\hline Gm21428 & -2.21 & unknown \\
\hline Ect2l & -2.25 & nucleotide exchange factor \\
\hline Dpt & -2.27 & ECM protein \\
\hline Prg4 & -2.28 & ECM molecule \\
\hline Prelp & -2.32 & ECM protein \\
\hline Olfr338 & -2.34 & olfactory receptor \\
\hline Angpt|7 & -2.42 & cell surface receptor \\
\hline Col10a1 & -2.50 & collagen \\
\hline Retnla & -2.54 & adipokine \\
\hline Myoc & -2.56 & ECM protein \\
\hline Thbs4 & -2.60 & ECM protein \\
\hline Pcolce2 & -2.75 & ECM protease \\
\hline Сyp2e1 & -2.77 & drug metabolism \\
\hline Cxcl13 & -2.83 & chemokine \\
\hline Clec3a & -2.87 & ECM protein \\
\hline Comp & -2.89 & ECM protein \\
\hline Ecrg4 & -3.21 & tumor supressor gene \\
\hline Cytl1 & -3.66 & cytokine \\
\hline
\end{tabular}

b

\begin{tabular}{|c|c|c|}
\hline gene & flight/ground & description \\
\hline Klhl38 & 3.52 & transferase \\
\hline Acot2 & 3.50 & thioesterase \\
\hline Fbxo32 & 3.37 & ubiquitin ligase \\
\hline Nr1d1 & 3.35 & circadian rhythm protein \\
\hline Trim63 & 3.22 & ubiquitin ligase \\
\hline Htra4 & 3.09 & serine peptidase \\
\hline Lox & 2.98 & lysyl oxidase \\
\hline Prg4 (lubricin) & 2.44 & ECM molecule \\
\hline Slc43a1 & 2.33 & solute carrier \\
\hline Lmod2 & 2.28 & actin binding protein \\
\hline Aldoc & 2.25 & aldolase \\
\hline Slc39a8 & 2.24 & solute carrier \\
\hline Etv5 & 2.23 & transcription factor \\
\hline$n-R 5 s 88$ & 2.22 & non-coding RNA \\
\hline Chac1 & 2.22 & glutathionine transferase \\
\hline Tango2 & 2.17 & golgi stress protein \\
\hline Pdk4 & 2.16 & pyruvate dehydrogenase kinase \\
\hline Chi3І1 & 2.11 & chitinase \\
\hline Impdh2 & 2.10 & inosine dehydrogenase \\
\hline Zfp600 & 2.08 & zinc finger protein \\
\hline Sesn1 & 2.08 & stress response gene \\
\hline Myf6 & 2.07 & myogenic factor \\
\hline Cfhr2 & 2.06 & complement factor \\
\hline Tsen15 & 2.05 & splicing endonuclease \\
\hline Omd & 2.03 & ECM protein \\
\hline Inmt & 2.03 & methyltransferase \\
\hline Sgcg & 2.02 & muscle glycoprotein \\
\hline Gm5886 & 2.02 & unknown \\
\hline Tacc2 & 2.02 & cell cycle protein \\
\hline Ankrd1 & 2.01 & ankryn-repeat protein \\
\hline Taf1d & -2.06 & transcription factor \\
\hline Hba-a2 & -2.07 & hemoglobin \\
\hline Mki67 & -2.09 & proliferation marker \\
\hline Slc4a1 & -2.09 & solute carrier \\
\hline Atp6v0d2 & -2.12 & vaculolar ATPase \\
\hline Sfrp2 & -2.13 & Wnt signalling \\
\hline Car1 & -2.13 & carbonic anhydrase \\
\hline Nr4a1 & -2.14 & nuclear receptor \\
\hline Mpo & -2.16 & myeloperoxidase \\
\hline Svs3b & -2.16 & secreted protein \\
\hline Hist1h3f & -2.17 & histone \\
\hline Retnlg & -2.19 & adipokine \\
\hline Hist1h3a & -2.19 & histone \\
\hline Top2a & -2.20 & DNA topoisomerase \\
\hline Hist1h3d & -2.22 & histone \\
\hline Hist1h3i & -2.26 & histone \\
\hline Hist1h2ab & -2.26 & histone \\
\hline Alpl & -2.36 & alkaline phosphatase \\
\hline Hist2h3c2 & -2.37 & histone \\
\hline $\mathrm{Hbb}-\mathrm{bs}$ & -2.43 & hemoglobin \\
\hline $\mathrm{Hbb}-\mathrm{bt}$ & -2.45 & hemoglobin \\
\hline Col1a1 & -2.49 & collagen \\
\hline Acp5 & -2.60 & acid phosphatase \\
\hline Hp & -2.62 & haptoglobin \\
\hline Car2 & -2.83 & carbonic anhydrase \\
\hline Igkv4-55 & -2.85 & immunoglobulin \\
\hline Gypa & -2.99 & glycophorin \\
\hline Ltf & -3.07 & growth factor signalling \\
\hline Igkv1-117 & -3.11 & immunoglobulin \\
\hline Ibsp & -3.38 & bone sialoprotein \\
\hline Mmp13 & -3.96 & metalloproteinase \\
\hline Mmp9 & -4.27 & metalloproteinase \\
\hline S100a9 & -4.70 & upregulate MMPs \\
\hline S100a8 & -6.41 & upregulate MMPs \\
\hline Ngp & -7.57 & peptidase inhibitor \\
\hline
\end{tabular}

Fig. 2 Cartilage RNA expression analysis. RNA isolated from articular cartilage a or sternal cartilage $\mathbf{b}$ were subjected to microarray analyses. Changes are expressed as fold change in flight compared to ground control samples. Only genes that are altered more than two-fold up in flight (shaded in green) and down in flight (shaded in orange) are listed. Structural cartilage extracellular matrix proteins or proteins associated with joint pathology are in bold 
AC fibrillation or fissure formation, and we conclude that exposure to microgravity results in moderate surface damage. Some chondrocytes were stained strongly for proteoglycan immediately surrounding the cell and may represent the production of new proteoglycan in the time elapsed (12-13 h) since returning to normal gravity although glycosaminoglycan synthesis assays are needed to confirm this.

Nine of the downregulated genes in spaceflight encode structural ECM components, including fibromodulin (fmod), osteoglycin (Ogn), osteomodulin (Omd), decorin (Dcn), dermatopontin (Dpt), PRELP (Prelp) collagen X (Col10a1), thrombospondin4 (Tsp4), and cartilage oligomeric matrix protein (COMP). Several other non-structural components with important cartilage ECM roles in development or osteoarthritis were also downregulated, including proteoglycan-related gene 4/lubricin (Prg4), procollagen C-endopeptidase enhancer 2 (Pcolce2), and Cytokine-like 1 (Cyt/1). Cytl1 is an autocrine factor that regulates chondrogenesis in mesenchymal cells ${ }^{12}$ and is required for cartilage homeostasis. ${ }^{13}$ Expression of Prg4 by superficial zone chondrocytes is acutely mechanosensitive; upregulation occurs with loading and associated with protection against osteoarthritis. ${ }^{14-17}$

The decrease in proteoglycan levels and downregulation of ECM molecules and genes that protect against osteoarthritic changes suggest that the early stages of cartilage breakdown are underway in the flight AC after 30 days of microgravity. However, since there is no evidence of significant collagen II degradation and the possible re-synthesis of proteoglycan in some chondrocytes, we suggest that cartilage recovery is possible and that 30 days of microgravity is insufficient for irreversible cartilage degradation.

In SC, there was no evidence of proteoglycan loss and a different suite of genes was altered in spaceflight. Of the downregulated genes, most notable were two prominent cartilage ECM-degrading enzymes: Mmp13 and Mmp9. This finding together with the downregulation of S100a8 and S100a9, which are known to stimulate $M m p$ gene activation in cartilage, ${ }^{18}$ suggest that less ECM proteolysis occurs in SF samples compared to non-flight samples in SC tissue.

The expression of several genes was altered in both cartilage tissues, including Prg4 and Omd, but opposite directions in the two cartilages. Osteomodulin regulates fibril diameter and is suggestive of new matrix synthesis in SC and reduced matrix production in AC. ${ }^{19} \mathrm{Prg} 4$ is chondroprotective and its upregulation in SC is further evidence that SC may be protected from degradation in microgravity.

Based on these differences in response to microgravity between the two cartilages, we suggest that the relative change in biomechanical environment determines the tissue response. During normal activities, AC is cyclically loaded with a significant fraction of body weight and then almost completely unloaded in microgravity. This change in loading triggers cartilage breakdown. In contrast, SC is loaded by cyclical lung expansion but does not experience the same magnitude of compressive loading as AC. Since the mice continue to breathe in microgravity and continuously load the tissue, the difference between mechanical loading in SF and controls is minimal, and cartilage breakdown is not initiated in SC.

Taken together, our findings suggest that maintenance of biomechanical loading during spaceflight will minimize $A C$ destruction.

\section{METHODS}

Animals

C57BL/6N male mice were flown for 30 days (477 Earth orbits) on the unmanned BION-M1 biosatellite between 19 April and 19 May 2013. ${ }^{20}$ Tissues were acquired from six male flight mice as part of NASA's Biospecimen Sharing Program. The mice were specific pathogen-free and
19-20 weeks old at the time of launch and start of control experiments. In addition to the six flight mice (SF), there were eight "flight" vivarium male ground control mice (SFV), seven asynchronous ground control males (GC), and seven asynchronous vivarium ground control males (GCV). Tissues were harvested $12-13 \mathrm{~h}$ post-landing. Flight and animal habitat details for the BION-M1 mission have been reported by Andreev-Andrievskiy et al. ${ }^{20}$

IACUC approval was obtained from the MSU Institute of Mitoengineering and of the Biomedical Ethics Commission of IBMP and the study was conducted in compliance with the European Convention for the Protection of Vertebrate animals used for Experimental and Other Scientific purposes.

\section{Tissue analyses}

Hindlimbs and elbow joints were dissected and right limbs placed in RNALater (Ambion) with left limbs placed in 10\% neutral-buffered formalin (NBF). Sternae with ribs attached were cut mid-sternum and the posterior half containing xiphoid process and two sternocostal joints placed in RNALater and the anterior half placed in NBF for histology. AC processing, toluidine blue histology and immunohistochemistry, were performed as previously reported. ${ }^{21}$ AC sections were stained for Safranin-O (Saf-O) and counterstained with hematoxylin using a protocol from the University of Rochester, Center for Musculoskeletal Research (https://www.urmc. rochester.edu/musculoskeletal-research/core-services/histology/protocols. aspx). Cartilage thickness measurements were taken along the tibial and femoral articular surfaces of Saf-O positive AC at 30 different sites for each section in the same relative position throughout the joint and included calcified and non-calcified regions together. These within-animal measurements were used to derive an average thickness for each animal. The thickness variation within an experimental group was similar to the average variation within individual samples (not shown).

For transmission electron microscopy, cartilage samples from SF and GC elbows were dissected from bone and post-fixed in $0.5 \%(\mathrm{v} / \mathrm{v})$ osmiumtetroxide and $1 \%(\mathrm{w} / \mathrm{v})$ potassium hexacyanoferrate (III) in $0.1 \mathrm{M}$ cacodylate buffer. After dehydration, specimens were incubated in propylenoxide and embedded in Epon. Ultrathin sections were cut, collected on copper grids, and negatively stained with $2 \%$ uranyl acetate. Electron micrographs were taken at $60 \mathrm{kV}$ with a Phillips EM-410 electron microscope (Ditabis, Pforzheim, Germany).

\section{RNA analyses}

RNALater-preserved AC from the femoral condyle and proximal tibia was dissected down to the calcified zone using an ophthalmic scalpel and tissue from SF and GC animals pooled separately. Half sternum pieces were dissected from ribs and SC dissected from bony segments and pooled. RNA was isolated using the MirVana RNA isolation kit (Life Technologies) and amplified by in vitro transcription with T7 RNA polymerase. Samples (100 ng) were then labeled using the Affymetrix GeneChip WT Plus protocol and hybridized to an Affymetrix Mouse Gene 1.0 ST GeneChip.

Analysis of microarray data was conducted using both Microarray Suite (MAS) version 5.0 and Robust Multi-Array Average (RMA). ${ }^{22}$ Differentially expressed genes were $\geq-2$ (decrease) or a fold change of $\geq 2$ (increase) with an FDR of 0.05 .

\section{Statistical analyses}

For the AC histological scoring analysis, measurements were taken from seven GC, seven GCV, five SF, and six SFV samples where good joint histology in the correct plane was obtained. Histology scoring for SC was conducted on six SF, seven GC, eight SFV, and seven GCV animals. Each animal received a single histology score representing the sum of separate histological parameters shown in Supplementary Table 1 with higher scores representing more overall cartilage degradation. Statistical differences between experimental groups (GC, GCV, SF, SFV) for total histological scores from each animal were examined using the Kruskal-Wallis test for multiple independent samples (http://astatsa.com/ KruskalWallisTest/). ${ }^{23}$ The omnibus $P$-value of 0.006 (d.f. $=3$ ) rejects the null hypothesis that all groups have the same distribution. Post-hoc pairwise multiple testing by Dunn was used to determine which pairs are different. ${ }^{24} P$-values were adjusted according to the family-wide error rate of Holm and then by the Benjamini-Hochberg method. ${ }^{25} P$-values for all pairwise comparisons used to calculate overall Kruskal-Wallis $P$-values are shown in Supplementary Table 2. Median, maximum score, minimum score, and 25th and 75th percentile of total histology data plotted for each experimental group are shown in Fig. $1 \mathrm{~d}$. 
Reporting Summary

Further information on experimental design is available in the Nature Research Reporting Summary linked to this article.

\section{DATA AVAILABILITY}

Microarray datasets generated and analyzed during the current study are available in the NASA GeneLab repository, https://genelab.nasa.gov/data/.

\section{ACKNOWLEDGEMENTS}

Microarray assays and data analysis were performed by the Gene Profiling Shared Resource at Oregon Health and Science University, Portland, OR, USA. This study was supported by NASA Grant \#NNX09AQ03G.

\section{AUTHOR CONTRIBUTIONS}

All authors made contributions to this research project including study design, data collection, and manuscript preparation.

\section{ADDITIONAL INFORMATION}

Supplementary information accompanies the paper on the npj Microgravity website (https://doi.org/10.1038/s41526-019-0063-6).

Competing interests: The authors declare no competing interests.

Publisher's note: Springer Nature remains neutral with regard to jurisdictional claims in published maps and institutional affiliations.

\section{REFERENCES}

1. Sanchez-Adams, J., Leddy, H. A., McNulty, A. L., O'Conor, C. J. \& Guilak, F. The mechanobiology of articular cartilage: bearing the burden of osteoarthritis. Curr. Rheumatol. Rep. 16, 451 (2014).

2. Buckwalter, J. A. \& Martin, J. A. Sports and osteoarthritis. Curr. Opin. Rheumatol. 16, 634-639 (2004)

3. Buckwalter, J. A. \& Martin, J. A. Osteoarthritis. Adv. Drug Deliv. Rev. 58, 150-167 (2006)

4. Kurz, B. et al. Pathomechanisms of cartilage destruction by mechanical injury Ann. Anat. 187, 473-485 (2005).

5. Scott, C. C. \& Athanasiou, K. A. Mechanical impact and articular cartilage. Crit. Rev. Biomed. Eng. 34, 347-378 (2006).

6. Kesikburun, S. et al. Comparison of intact knee cartilage thickness in patients with traumatic lower extremity amputation and nonimpaired individuals. Am. J. Phys. Med. Rehabil. 94, 602-608 (2015).

7. Vanwanseele, B., Eckstein, F., Knecht, H., Spaepen, A. \& Stussi, E. Longitudinal analysis of cartilage atrophy in the knees of patients with spinal cord injury. Arthritis Rheum. 48, 3377-3381 (2003)

8. Vanwanseele, B., Eckstein, F., Knecht, H., Stussi, E. \& Spaepen, A. Knee cartilage of spinal cord-injured patients displays progressive thinning in the absence of normal joint loading and movement. Arthritis Rheum. 46, 2073-2078 (2002).
9. Carmeliet, G., Vico, L. \& Bouillon, R. Space flight: a challenge for normal bone homeostasis. Crit. Rev. Eukaryot. Gene Expr. 11, 131-144 (2001).

10. Ilyin, E. \& Organov, V. Microgravity and musculoskeletal system of mammals. Adv. Sapce Res. 9, 11-19 (1989).

11. Buckwalter, J. A. Articular cartilage injuries. Clin. Orthop. Relat. Res. 402, 21-37 (2002).

12. Kim, J. S., Ryoo, Z. Y. \& Chun, J. S. Cytokine-like 1 (Cytl1) regulates the chondrogenesis of mesenchymal cells. J. Biol. Chem. 282, 29359-29367 (2007).

13. Jeon, J. et al. Cytokine-like 1 knock-out mice (Cytl1-/-) show normal cartilage and bone development but exhibit augmented osteoarthritic cartilage destruction. J. Biol. Chem. 286, 27206-27213 (2011).

14. Abusara, Z. et al. Muscular loading of joints triggers cellular secretion of PRG4 into the joint fluid. J. Biomech. 46, 1225-1230 (2013).

15. Nugent, G. E. et al. Static and dynamic compression regulate cartilage metabo lism of PRoteoGlycan 4 (PRG4). Biorheology 43, 191-200 (2006).

16. Nugent-Derfus, G. E. et al. Continuous passive motion applied to whole joints stimulates chondrocyte biosynthesis of PRG4. Osteoarthr. Cartil. 15, 566-574 (2007).

17. Ogawa, H., Kozhemyakina, E., Hung, H. H., Grodzinsky, A. J. \& Lassar, A. B. Mechanical motion promotes expression of Prg4 in articular cartilage via multiple CREB-dependent, fluid flow shear stress-induced signaling pathways. Genes Dev. 28, 127-139 (2014).

18. Zreiqat, H. et al. S100A8 and S100A9 in experimental osteoarthritis. Arthritis Res. Ther. 12, R16 (2010).

19. Tashima, T., Nagatoishi, S., Sagara, H., Ohnuma, S. \& Tsumoto, K. Osteomodulin regulates diameter and alters shape of collagen fibrils. Biochem. Biophys. Res. Commun. 463, 292-296 (2015).

20. Andreev-Andrievskiy, A. et al. Mice in Bion-M 1 space mission: training and selection. PLoS ONE 9, e104830 (2014).

21. Fitzgerald, J. et al. Evidence for articular cartilage regeneration in MRL/MpJ mice. Osteoarthr. Cartil. 16, 1319-1326 (2008).

22. Irizarry, R. A. et al. Summaries of Affymetrix GeneChip probe level data. Nucleic Acids Res. 31, e15 (2003).

23. Kruskal, W. H. \& Wallis, W. A. Use of ranks in one-criterion variance analysis. JASA 47, 583 (1952)

24. Dunn, O. J. Multiple comparisons among means. JASA 56, 10 (1961).

25. Benjamini, Y. \& Hochberg, Y. Controlling the false discovery rate: a practical and powerful approach to multiple testing. J. R. Stat. Soc. Ser. B (Methodol.) 57, 12 (1995).

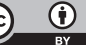

Open Access This article is licensed under a Creative Commons Attribution 4.0 International License, which permits use, sharing, adaptation, distribution and reproduction in any medium or format, as long as you give appropriate credit to the original author(s) and the source, provide a link to the Creative Commons license, and indicate if changes were made. The images or other third party material in this article are included in the article's Creative Commons license, unless indicated otherwise in a credit line to the material. If material is not included in the article's Creative Commons license and your intended use is not permitted by statutory regulation or exceeds the permitted use, you will need to obtain permission directly from the copyright holder. To view a copy of this license, visit http://creativecommons. org/licenses/by/4.0/.

(c) The Author(s) 2019 